\title{
SMA observations towards massive clouds in the central molecular zone
}

\author{
Xing Lu ${ }^{1,2}$, Qizhou Zhang ${ }^{1}$, Jens Kauffmann ${ }^{3}$ and Thushara Pillai ${ }^{3}$ \\ ${ }^{1}$ Harvard-Smithsonian Center for Astrophysics, 60 Garden Street, Cambridge, MA 02138, USA \\ email: xlu@cfa.harvard.edu \\ ${ }^{2}$ School of Astronomy \& Space Science, Nanjing University, Nanjing 210093, Jiangsu, China \\ ${ }^{3}$ California Institute of Technology, Astronomy Department, 1200 East California Blvd., \\ Pasadena, CA 91125, USA
}

\begin{abstract}
Recently we conducted a mini-survey towards a sample of six massive clouds with surface density $>10^{24} \mathrm{~cm}^{-2}$ in the central molecular zone (CMZ) of the Milky Way, with the SMA at $280 \mathrm{GHz}$ in the compact array and at $230 \mathrm{GHz}$ in the compact/subcompact arrays. The data reveal compact dust continuum peaks, some of which are also associated with organic molecular lines and thermal $\mathrm{SiO}$ emission. The subcompact array data helps recover more structures, e.g. the regularly spaced, well-aligned continuum fragments in the $20 \mathrm{~km} \mathrm{~s}^{-1}$ cloud. Shock tracers such as $\mathrm{SiO}$ are found in all the clouds. Our observations suggest potential protostellar origin for some of the dust continuum peaks in these regions.
\end{abstract}

The inner $500 \mathrm{pc}$ of our Galaxy, known as the central molecular zone (CMZ), is a star-forming environment with very extreme physical properties. Despite its large reservoir of dense gas that could form massive stars and clusters similar to the starbursts seen in galaxies, the CMZ appears to have an unusually low star formation rate. One possibility is that a deeply embedded protostellar population has been missed by existing observations.

Kauffmann et al. (2013) used the SMA $280 \mathrm{GHz}$ dust continuum and $\mathrm{N}_{2} \mathrm{H}^{+}$lines to map the dense gas in one of the CMZ clouds, G0.253+0.016, and found that despite its large mass measured by single-dish observations, little dense gas $\left(>10^{5} \mathrm{~cm}^{-3}\right)$ is present in the high angular resolution interferometric observations, which may explain the low level of star formation. We then expanded our observations to the other five clouds, including Sgr D, Sgr B1 off, $50 \mathrm{~km} \mathrm{~s}^{-1}$ cloud, $20 \mathrm{~km} \mathrm{~s}^{-1}$ cloud, and Sgr C, at $280 \mathrm{GHz}$ with the SMA in its compact array. We are also in the process of surveying all the six clouds at $230 \mathrm{GHz}$ in both the compact and subcompact arrays, to use the abundant molecular lines in this band to identify potential star forming sites.

\section{Dust continuum peaks in the clouds}

In the $280 \mathrm{GHz}$ data, we found abundant dust continuum structures in all the clouds except G0.253+0.016, while $\mathrm{N}_{2} \mathrm{H}^{+} J=((3-2)$ emission which also traces dense gas is widely found. In the $230 \mathrm{GHz}$ SMA observations, in addition to compact array data, we obtained sub-compact array data which provide shorter baselines, in order to recover extended flux. The continuum emission retrieved from the combination of the two arrays (Figure 1) shows more sub-structures than the $280 \mathrm{GHz}$ data. For example, in the 20 $\mathrm{km} \mathrm{s}^{-1}$ cloud, we found that five continuum peaks are regularly spaced and well-aligned, similar with the molecular core fragments found in Galactic surveys (e.g. Jackson et al. 2010). Based on the velocity of the dense gas tracer $\mathrm{H}_{2} \mathrm{CO}$, the five peaks present a 


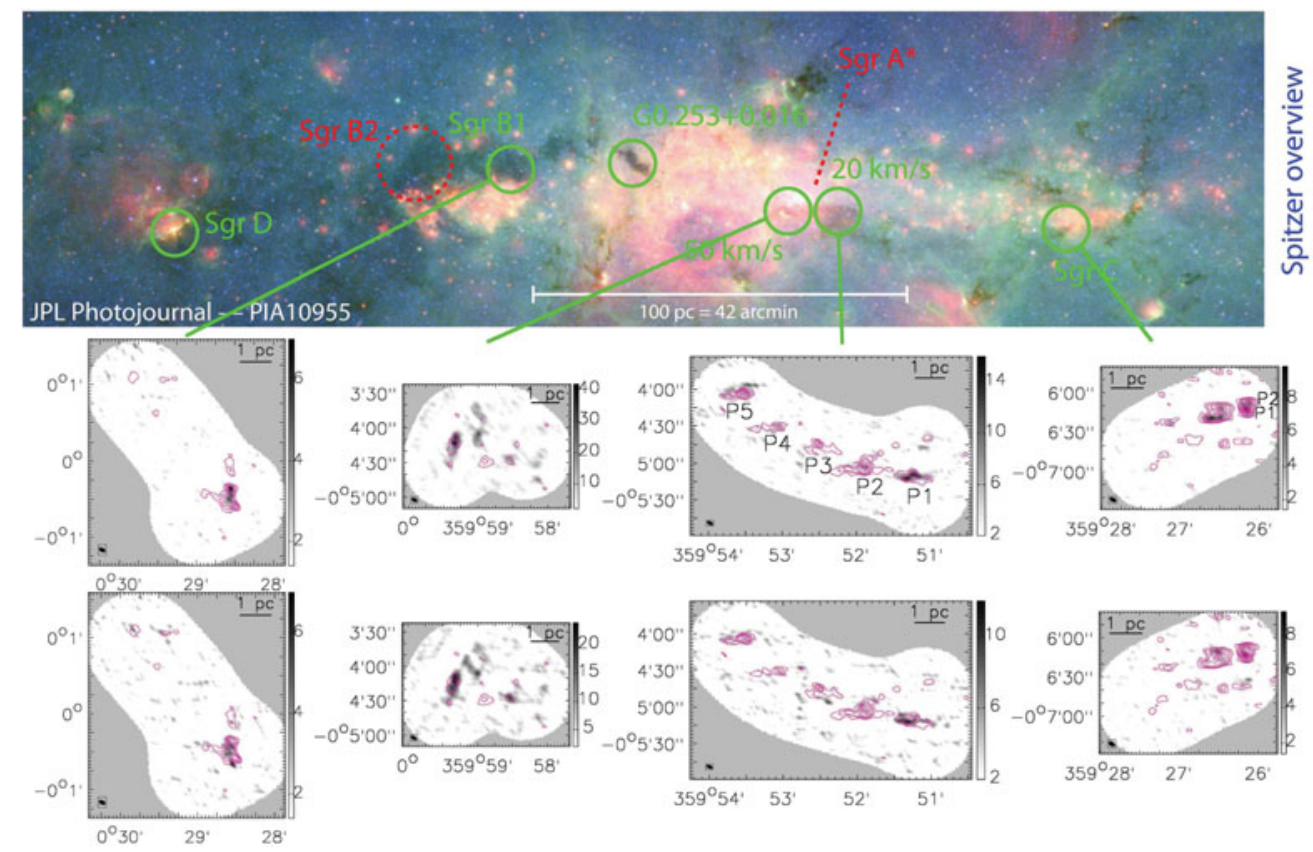

Figure 1. An overview of the CMZ and the $230 \mathrm{GHz}$ SMA observations. Upper panel: Spitzer 3-color image of the CMZ (red: $24 \mu \mathrm{m}$; green: $8 \mu \mathrm{m}$; blue: $3.6 \mu \mathrm{m}$ ). The sources included in our surveys are marked by green circles. Lower panels: Combined compact/subcompact SMA $230 \mathrm{GHz}$ data of four CMZ clouds, in galactic coordinates. The observation of other two clouds (Sgr D and G0.253+0.016) is in progress. The upper images show the integrated intensity (in unit of Jy/beam) of $\mathrm{CH}_{3} \mathrm{OH} 8(-1,8)-7(0,7)$ transition line. The contours are the $230 \mathrm{GHz}$ continuum, in increments of $[3,6,9,15,25,35]^{*} \mathrm{RMS}$, where $\mathrm{RMS}=5 \mathrm{mJy} / \mathrm{beam}$. The lower images show the integrated intensity of $\mathrm{SiO} J=(5-4)$ with identical contours. [A COLOR VErsion is aVAilable online.]

velocity gradient from blueshifted to redshifted, from P1 to P5. If the cloud is on the near side of the CMZ, it may indicate an infalling trend along the filamentary structure towards Sgr A*.

\section{Potential star forming sites}

The dust continuum peaks in Sgr C (P1 and P2 in Figure 1) have been found to be protostellar in nature (Kendrew et al. 2013). Among the five dust continuum peaks in the $20 \mathrm{kms}^{-1}$ cloud (see Figure 1), P2 seems to be a potential star forming site as well. Assuming a temperature of $30 \mathrm{~K}$ and an emissivity index of 1.5, the mass derived from the $230 \mathrm{GHz}$ dust emission is $\sim 1300 \mathrm{M}_{\odot}$ at a distance of $8.4 \mathrm{kpc}$. P2 presents strong $\mathrm{N}_{2} \mathrm{H}^{+}$emission, as well as spectral lines of shock tracers such as $\mathrm{SiO}$ and HNCO. Centimeter continuum emission (Betsy Mills, private communication) and water masers (Sjouwerman et al. 2002) have been found around this peak. After we analyze the kinematics using dense gas tracers, we will determine whether it is gravitationally bound. We plan to analyze the entire sample in the same manner and identify protostars in all six clouds. 


\section{References}

Kauffmann, J., Pillai, T., \& Zhang, Q. 2013, ApJ 765, L35

Jackson, J. M., Finn, S. C., Chambers, E. T., et al. 2010, ApJ 719, L185

Kendrew, S., Ginsburg, A., Johnston, K., et al. 2013, ApJ 775, L50

Sjouwerman, L. O., Lindqvist, M., van Langevelde, H. J., et al. 2002, A\&\&A 391, 967 